\title{
Routing Strategy Selection for Zigbee Mesh Networks
}

\author{
Ramanathan Karthikeyan \\ Department of Electronics \& Communication Engineering, Kumaraguru College of Technology, \\ Coimbatore, India \\ E-mail: karthikeyanrece@gmail.com \\ Received April 29, 2010; revised May 31, 2010; accepted July 3, 2010
}

\begin{abstract}
Based on IEEE 802.15.4 Low Rate-Wireless Personal Area Network (LR-WPAN) standard, the Zigbee standard has been proposed to interconnect simple, low rate and battery powered wireless devices. The deployment of Zigbee networks is expected to facilitate numerous applications such as Home-appliance networks, home healthcare, medical monitoring and environmental sensors. An effective routing scheme is more important for Zigbee mesh networks. In order to achieve effective routing in Zigbee Mesh networks, a Zigbee protocol module is realized using NS-2. The suitable routing for different data services in the Zigbee application layer and a best routing strategy for Zigbee mesh network are proposed. The simulation shows the selection of suitable routing for continuous data services and for bursting data services in the Zigbee application layer and the comparison of three routing strategies namely ERD (All packets Enable Route Discovery), SRD (All packets Suppress Route Discovery) and BOS (routing Based on Data Services) with respect to efficiency and overhead.
\end{abstract}

Keywords: LR-WPAN, NS-2, ERD, SRD, BOS

\section{Introduction}

Zigbee is an emerging worldwide standard for Wireless Personal Area Networks (WPAN). Under the main goal to provide low-power, cost effective, flexible, reliable and scalable wireless products Zigbee Alliance has been developing and standardizing the Zigbee network. Based on IEEE 802.15.4 [1], Zigbee defines three types of devices. They are Zigbee Coordinator, Zigbee Router and Zigbee End device. Zigbee networks support star, tree and mesh topologies, self-forming and self-healing as well as more than 65000 address spaces; thus the network can be easily extended in terms of size and coverage area. The star topology of Zigbee is mainly designed for the simple communication from one node to several nodes. The tree network uses a hierarchical tree routing mechanism. The mesh network uses the mixed routing method combined with Z-AODV and hierarchical tree routing.

\section{Zigbee Routing Algorithms}

There are two routing algorithms in Zigbee network layer. They are modified Ad Hoc on Demand Distance Vector (Z-AODV) and Hierarchical Routing algorithms [2].

\subsection{Z-AODV}

Currently AODV [3] is the easiest and most widely implemented MANET protocol. Z-AODV is one of the earliest AODV simplified versions. Z-AODV removes the following items from the AODV specification such as Sequence number, gratuitous RREP, hop count, Hello message, precursor limits. In Z-AODV if the communications are unidirectional, the destination sends connect message to the source. If data traffic is bidirectional, no additional messages are used. In any case, a source detects a link break in a route when it doesn't receive messages from the destination.

\subsection{Hierarchical Routing Algorithm}

The hierarchical routing algorithm depends on the topology and a distributed addressing scheme of Zigbee networks. There are three types of devices in Zigbee networks. They are Coordinator, Router and End device. A Zigbee coordinator is responsible for initializing, maintaining and controlling the network. A star network has a coordinator with end devices directly connecting to the coordinator. For tree and mesh networks, Zigbee devices can communicate with each other in multihop 
fashion. The network is formed by one Zigbee coordinator and multiple Zigbee routers. A device can join a network as an end device by associating with a coordinator or a router [4].

Before forming a network, the coordinator determines the maximum number of children of a router $\left(C_{m}\right)$, the maximum number of child routers of a router $\left(R_{m}\right)$, and the depth of the network $\left(L_{m}\right)$ [5]. A child of a router can be a router or an end device, so $\left(C_{m} \geq R_{m}\right)$. Zigbee specifies a distributed address assignment using parameters $C_{m}, R_{m}$ and $L_{m}$ to calculate the nodes network addresses. In Zigbee if a device joins a network successfully, it can obtain a network address from the coordinator or a router. The basic idea of the assignment is that for the coordinator and the routers in every layer, the whole address space is logically partitioned into $R_{m}+1$ block. The first $R_{m}$ blocks are to be assigned to the router child devices and the last block is reserved for the $\left(C_{m}-R_{m}\right)$ child end devices. In order to make the assignment easily, a function Cskip can be computed by $C_{m}, R_{m}$ and $L_{m}$. The value of this function is the size of address sub-block being distributed by each parent at the depth of its router child devices for a given network depth $d$.

$$
\text { Cskip }(d)= \begin{cases}1+C_{m}\left(L_{m}-d-1\right), & \text { if } R_{m}=1 \\ \frac{1+C_{m}-R_{m}-C_{m} R_{m}{ }^{L_{m}-d-1}}{1-R_{m}}, & \text { otherwise }\end{cases}
$$

$A_{n}$ is computed by the following formula.

$$
A_{n}=A_{\text {parent }}+\operatorname{Cskip}(d-1) R_{m}+n
$$

Here $A_{\text {parent }}$ denotes the address of the parents, $n$ denotes the $n^{\text {th }}$ end device, and $1 \leq n \leq\left(C_{m}-R_{m}\right)$. When Zigbee adopted the hierarchical routing algorithm and a device called $X$ with address $A$ and depth $d$ received a packet, the device extracted the destination address called $D$.

$$
N=\left\{\begin{array}{l}
D, \text { if } D>A+R_{m} \times \operatorname{Cskip}(d) \\
A+1+\left[\frac{D-(A+1)}{\operatorname{Cskip}(d)}\right] \times \operatorname{Cskip}(d), \text { Otherwise }
\end{array}\right.
$$

If $D>A+R_{m} \times C \operatorname{skip}(d)$, the destination is the direct descendent of $X$, and $X$ forward the packet to this direct descendent. If not, the destination is the indirect descendents of $X$, so $X$ forward the packet to one of its child with address computed by

$$
A+1+\left\lfloor\frac{D-(A+1)}{\operatorname{Cskip}(d)}\right\rfloor \times \operatorname{Cskip}(d) .
$$

In this way the network address is assigned to the network elements in the Zigbee network using hierarchical routing.

\section{Simulated Results}

\subsection{Performance of Tree Routing and Z-Aodvrouting over Zigbee Networks}

A Zigbee protocol module is developed using NS-2 with the following specifications. The simulation area is $50 \times$ $50 \mathrm{~m}^{2}$, the number of nodes is 21 , Transmission range is $12 \mathrm{~m}$, Packet error ratio is $0.2 \%$, Data rate is $250 \mathrm{Kbps}$, Packet size is 70 bytes and Simulation time is $150 \mathrm{sec}$.

From Figure 1 we observe that, the tree routing has faster response in forwarding the data packets since it doesn't need to initiate the routing tables. The flow starts at $25^{\text {th }}$ second. At $27^{\text {th }}$ second, 7 tree routed data packets are arrived at the destination node. The Z-Aodv routed data packets arrived at $27^{\text {th }}$ second only because Z-Aodv must initiate the routing discovery. Z-Aodv after establishing its routing table the number of data packets in two routing methods will tend to be the same. Z-Aodv always chooses the route with less number of hops and the tree routing usually won't obtain shorter route. So the data frames transmitted in tree routing network are always 1.2 to1.4 times more than the data frames transmitted in Z-Aodv network. So the tree routing of Zigbee is suitable for bursting data transmissions and Z-Aodv is suitable for continuous data transmissions.

\subsection{Comparison of Three Routing Strategies for Zigbee Mesh Network}

A data flow consists of mixture of continuous data and bursting data is used in this simulation. The Zigbee mesh network uses a mixed routing mechanism combined with tree routing and $\mathrm{Z}$-Aodv routing [6].

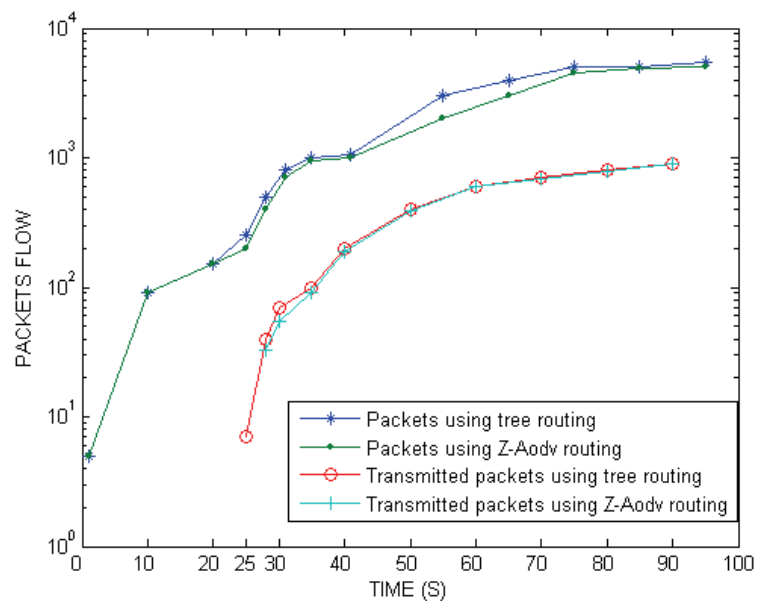

Figure 1. Performance comparison of tree routing and Z-AODV routing. 
The Discover Route Field in the header of the data frames decides the routing approach for the data frames. If it has the value of Suppress Route Discovery, it uses the routing tables that exist already. When there is no corresponding address of the destination node the network will use tree routing.

If the Discover Route Field has the value of Enable Route Discovery and when the routing address is there in the routing table, the routing will follow this routing table. Otherwise the router will initiate the routing discovery. When the node has no ability to initiate the routing discovery, it will use tree routing. Based on the previous section simulation results, we can choose the binding data services in the Zigbee application layer will always use Enable Route Discovery routing method and the bursting data services in the Zigbee application layer will always use the Suppress Route Discovery routing method. This kind of routing method is called as routing based on data services. Efficiency is defined as the ratio between the transmitted data bytes and the total transmitted bytes. Figure 2 shows the comparison of three routing strategies with respect to efficiency.

The efficiency of SRD routing method is the highest. The efficiency of ERD routing method is the lowest. BOS has to initiate the routing discovery for continuous data flow alone. ERD has to initiate the routing discovery for both continuous and bursting data flow. The increase of control overhead makes the efficiency of ERD low.

Figure 3 shows the average number of frames required to transmit a single data packet in all the three routing strategies for different bursting data conditions. SRD requires more frames to transmit a single data packet, since it uses the defined tree path. But ERD elects the shortest path and in turn it requires fewer

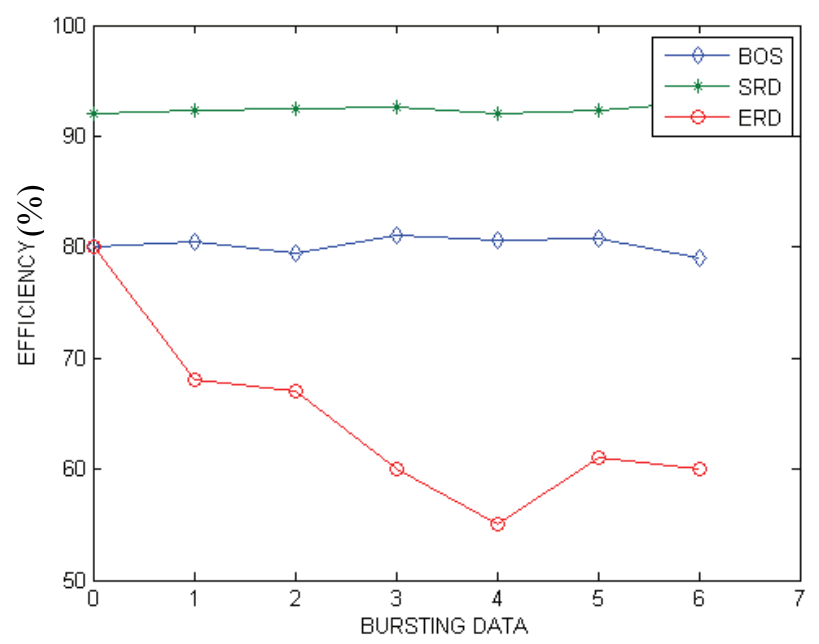

Figure 2. Comparison of ERD, SRD \& BOS in the aspect of Efficiency.

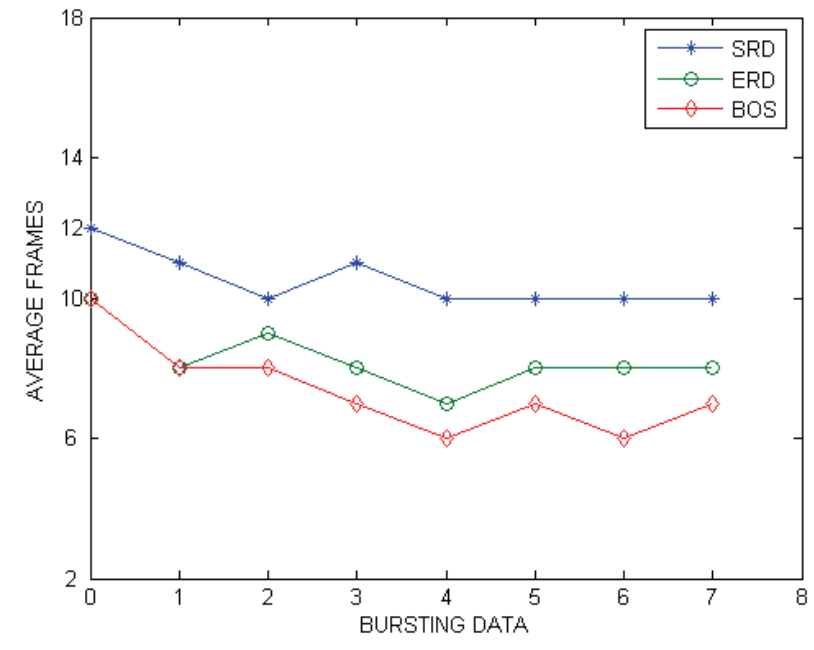

Figure 3. Average number of frames required to transmit a data packet in ERD, SRD \& BOS.

frames. The BOS routing method cuts down the consumption of routing discovery for bursting data since it uses tree routing. So it has the least overhead. Comparatively BOS has the least overhead than ERD and SRD, which accordingly reduce the power consumption.

Thus in turn it is more suitable and much beneficial for Low Power IEEE 802.15.4 \& Zigbee.

\section{Conclusions}

In this paper, the selection of suitable routing for continuous data services and for bursting data services in the Zigbee application layer is proposed. The three routing strategies namely Enable Route Discovery(ERD), Suppress Route Discovery(SRD), and routing Based On data Services(BOS) are compared in the aspects of Efficiency, overhead and BOS is proposed as the suitable routing strategy for Zigbee mesh networks.

\section{References}

[1] IEEE. Std. 802.15.4, "Wireless Medium Access Control (MAC) and Physical Layer (PHY) Specifications for Low Rate Wireless Personal Area Networks," 2003.

[2] J. Sun, Z. X. Wang and H. Wang, "Research on Routing Protocols Based on Zigbee Network," Proceedings of the Third International Conference on Intelligent Information Hiding and Multimedia Signal Processing, Kaohsiung, Taiwan, November 26-28, 2007, pp. 639-642.

[3] C. Perkins and E. B. Royer, "Ad Hoc On-demand Distance Vector(AODV) Routing,” RFC 3561, July 2003.

[4] T. Kim, D. Kim and S. Yoo, "Shortcut Tree Routing in Zigbee Networks," In $2^{\text {nd }}$ International Symposium on Wireless Pervasive Computing, San Juan, Puerto Rico, February 5-7, 2007, pp. 42-47. 
[5] Zigbee Alliance, "Zigbee Specification Version 1.0," December $14^{\text {th }}, 2004$. http://www.zigbee.org

[6] X. H. Li and K. L. Fang, "An Improved Zigbee Routing Strategy for Monitoring Systems," IEEE Proceedings of
First International Conference on Intelligent Networks and Intelligent Systems, Wuhan, 2008, pp. 255-258. 